\title{
ANALYSIS OF CLUSTER DIMENSION AND MORPHOLOGY OF ROOM TEMPERATURE GROWN NANOCARBON USING CATHODIC ARC AND ITS INFLUENCE ON FIELD ASSISTED ELECTRON EMISSION
}

\author{
Niranjana .S ${ }^{1}$, Shounak De $^{2}$, Moorthy .V.H.S ${ }^{3}$, Niranjan .U.C ${ }^{4}$, Satyanaryana .B.S ${ }^{5}$ \\ ${ }^{1}$ Department of Biomedical Engineering, ${ }^{2,3,45}$ Department of Electronics and Communication Engineering, \\ Manipal Institute of Technology, Manipal, India \\ E-mail: 'niranjana.s@manipal.edu
}

\begin{abstract}
Low temerpatuere grown nanocluster carbon thin films grown using a continuous cathodic arc process at room temperature are found to be interesting material for wide range of and nanoelectronic application. Reported is the study of the influence of background gases such as helium and nitrogen on the nanoclsuter dimension and morphology and a possible influence of the same to field assisted electron emission properties. The Helium partial pressure was changed from $5 \times 10^{-4}$ to 50 Torr under two different nitrogen partial pressure conditions namely $1 \times 10^{-4}$ Torr and $1 \times 10^{-3}$ Torr respectively. Raman and SEM data for these samples were used to estimate the cluster dimension structural and morphological properties. The surface morphology of the films grown, varied from atomic smooth surface to clusters with varying sizes (50-200 nm). A methodology for the estimation of cluster dimension from the Raman data was established and the estimated data compared with dimensions derived from SEM images. Effort was made to look at the possible correlation of between process parameters, estimated parameters and field assisted electron emission data. The curve fitting analysis and the observed correlation, indicates possibility of establishing Raman Spectroscopy as an instantaneous characterization approach for the evaluation of nanocarbons.
\end{abstract}

Key words: Morphology, Cathodic arc Process, Cluster dimension,

\section{INTRODUCTION}

Carbon based nanotechnology began with discovery of Fullerenes in 1985[1, 2] and the carbon nanotubes in 1991 [1,2]. The advances in Very Large Scale Integrated Circuits (VLSI) process technologies, the advent of new materials and the of miniaturization of systems leading to Micro / Nano Electro Mechanical System (MEMS / NEMS) has led innovators to move towards System on Chip (SoC) and System in Package (SiP) based applications. The new miniaturized technology demands the self aligned nanomaterials which are expected to be the building blocks of future nanoelectronic devices [1-5]. Among the many nanomaterials, nanocarbon is one of the most studied material, in its various manifestations include nanodiamond, single-walled / multi-walled carbon nanotubes (SWNT/MWNT), fullerenes $\left(\mathrm{C}_{60}\right)$, nanohorns, nanowalls, nanowires, nanofibers, nanocluster or nanostructured carbon. Growing areas of technology were these nanocarbon find applications include Surface Engineering, Nanoelectronics, Vacuum nanoelectronics, Sensors, Biomedical applications, Novel energy sources, Interconnects in ICs, Novel light, strong and even conducting Composite materials and Flexible electronics [1-3,5-7].

Innumerable process technologies have been used to grow nanocarbon based materials. Some of the more common ones include

Thermal Chemical Vapor Deposition (TCVD), Hot Filament CVD (HFCVD), Plasma CVD Microwave CVD,
Pulsed Laser Ablation and Cathodic arc. The nanocarbon film's structural, compositional and morphological properties are highly dependent on the process parameter such as temperature, pressure, compositional gas ratios and ion energy[12,16]. Further most of the reported nanocarbon films have been grown at relatively high temperatures. $\left(700-1000^{\circ} \mathrm{C}\right)$.

The Cathodic arc process is of interest because it is an evolving technology. Cathodic arc has already been demonstrated as suitable for applications in areas like tribology, low K dielectrics and conformal metal coating for Very Large Scale Integration(VLSI)/ULSI. However it is yet to be proven as a suitable technology for electronic materials $[13,18,19]$. However recently there are a few reports on electronic applications like field assisted electron emission. This process is of great interest because it offers great opportunity for tailoring nanocarbon based materials over a wide range of properties[5]. Thus the growth of low temperature grown nanocarbon using cathodic arc based system could be interesting for flexible electronics or electronics on glass or plastic. The growth of thin film could be influenced by variety of process parameters including substrate temperature, substrate material type, gases involved in the process, deposition rate, mode of arcing (continuous or pulsed), arc current and arc voltage [9].

The understanding of nanocarbons films can be established only when it is fully characterized for all it's structural, compositional, morphological, electronics and optoelectronic properties. However in the case of 
nanocarbons it is even more complicated unlike a material like silicon, because the carbon atoms can have different bonding. It could be a diamond like bonding $\left(\mathrm{sp}^{3}\right)$ or a graphite like bonding $\left(s p^{2}\right)$ or a polymeric bond $\left(s p^{1}\right)$. Further the material may have a mixed phase of bonds. The characterization techniques and more importantly the analysis or interpretation of the data for such complex mixed phase material is not yet well understood and hence not established. More importantly while looking for bonding composition and nano dimensional features. Among many techniques Raman Spectroscopy offers the option of a nondestructive, instantaneous characterization [18-20]. Reported is a study and analysis of room temperature grown nanocluster carbon using with Raman and SEM. Also reported is the correlation between the derived compositional and nano dimensions parameters with the earlier reported field assisted electron emission behavior $[8,10,11,14]$ from these room temperature grown nanocluster carbon. Thus establish the viability of nano dimension and composition estimation of mixed phase nano carbons using Raman spectroscopy.

\section{EXPERIMENT}

Nanocluster Carbon films were deposited in a Cathodic Vacuum Arc system at room temperature in the presence of background gases such as helium, hydrogen and nitrogen. The chamber was first evacuated to a vacuum of $\sim 10^{-7}$ Torr, before initiating the deposition process. Nanocluster carbon films were deposited on $\left(\mathrm{n}^{+}\right)$ silicon and glass substrates. No substrate bias was applied. The films were typically grown to a thickness of $150-200 \mathrm{~nm}$. The films were grown under $10^{-3}$ Torr and $10^{-4}$ Torr Nitrogen partial pressure. The helium concentration was varied from $5 \times 10^{-4}$ to 50 Torr (wrong every were the unit0.05 to 50 Pascal. The SEM images were taken using a JEOL FESEM. The Raman measurements were carried out using a Reinshaw Raman spectroscopy equipment with a 514.5nm excitation source. The samples for Raman measurements were deposited on silicon and glass substrates.

\section{EXPERIMENTAL RESULTS AND DISCUSSION}

The morphological and dimensional features of various nanocluster carbon were studied using Scanning Electron Microscopic images (SEM). Shown in figure $1(a, b \& c)$ are the typical SEM images of the various nanocluster carbon thin films grown under various helium and nitrogen concentration. The surface morphology images of the samples show the variation of nanocluster size from atomic smooth surface to varying dimensions of clusters and also nanofibers in in the measured range of process parameters mentioned earlier.
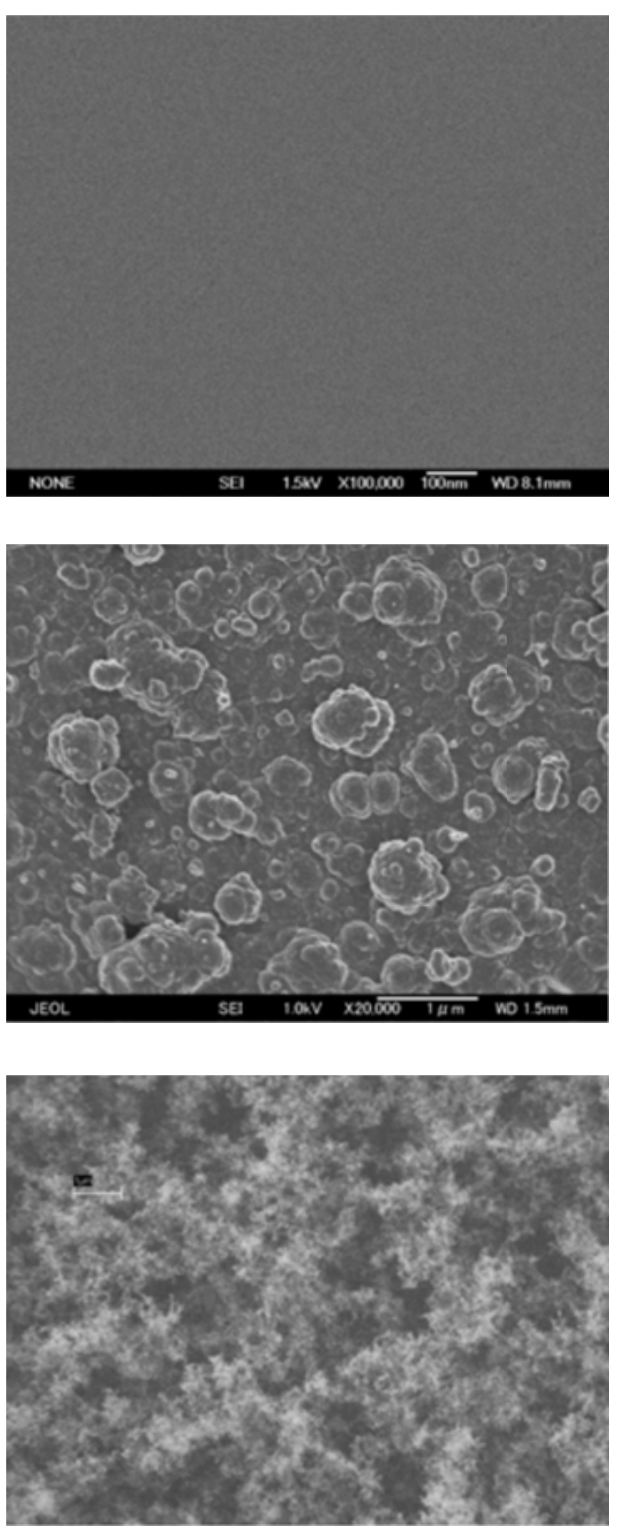

C

Fig 1, SEM image of nanocarbon thin films grown using cathodic arc process at room temperature with varying surface morphology (a) Near atomic smooth surface, (b) Nano Clusters and @ Nanofibers.

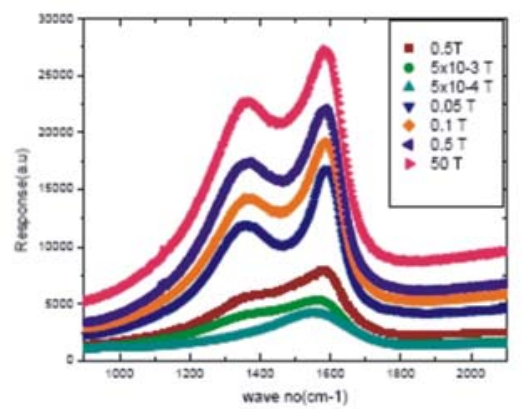

Fig 2. Raman response curve for various nanocaluster carbon under varying Helium Partial pressure conditions . 


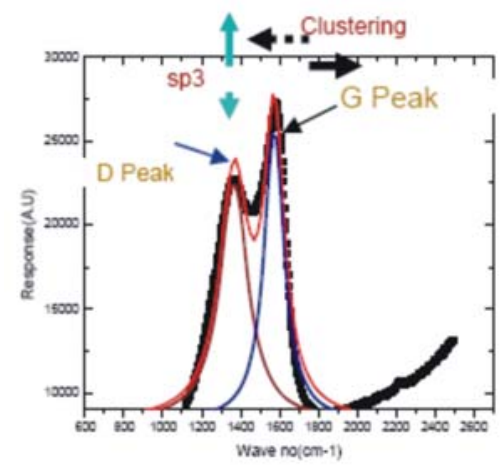

Fig 3. Typical Raman Response based model of nanocarbon and deconvolved $\mathrm{G}$ and $\mathrm{D}$ peaks

The SEM images of nanocluster carbon grown using cathodic arc system[10] shown in figure 1 clearly shows the possibility to grow carbon films with surface morphology varying from a)atomic smooth surface film (low nitrogen concentration $\left[<10^{-5} \mathrm{Torr}\right]$ ) to clusters of varying dimensions ( nitrogen $=10^{-3}$ torr and helium 0.05 Torr ) even fibers ( nitrogen $=10^{-3}$ torr and helium 50 Torr ). The cluster dimension varied from 50 to $200 \mathrm{~nm}$. It was observed that the clustering is clearly influenced by both helium partial pressure and nitrogen concentration has influence on the properties.

Shown in Fig.2 are the Raman Response of nanocluster carbon films measured under variying helium partial pressures $\left(5 \times 10^{-3}\right.$ to 50 Torr $)$ at two different $10^{-4} \mathrm{Torr}$ and $10^{-3}$ Torr nitrogen partial pressure condition. Further they were analyzed using curve fitting approach with lorenzian deconvolution technique. Shown in figure 3 is the typical deconvolved $D$ peak and $G$ peak derived from curve fitting technique. Also $\mathrm{G}$ peak shift towards right or left indicates the variation of cluster dimension as well its properties . The extracted parameters include: the ratio of the intensity of the $D$ peak $\left(I_{d}\right)$ to that :of the intensity of $G$ peak $\left(I_{g}\right)$ as $I_{d} / l g$ ratio, Full Width Half Maximum(FWHM), FWHM ratio, area of $G$ peak, area of $D$ peak $G$ peak position and $D$ peak position.For more detailed information from the Raman response the $G$ peak area were analyzed.

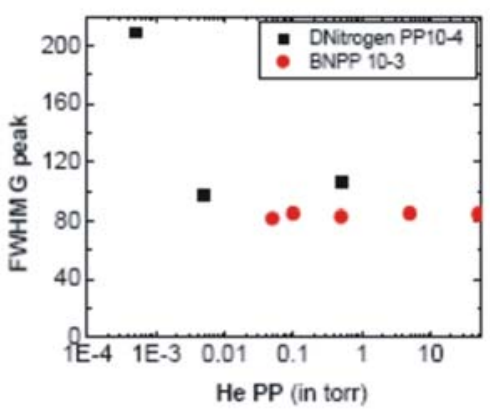

Fig 4: Variation of FWHM of Gpeak derived from Raman response for nanocluster.
Shown in Fig. 4, is the variation of FWHM of G peak derived from Raman response for nanocluster samples grown with nitrogen partial pressure $10^{-3}$ and $10^{-4}$ Torr with variation in Helium partial pressure.
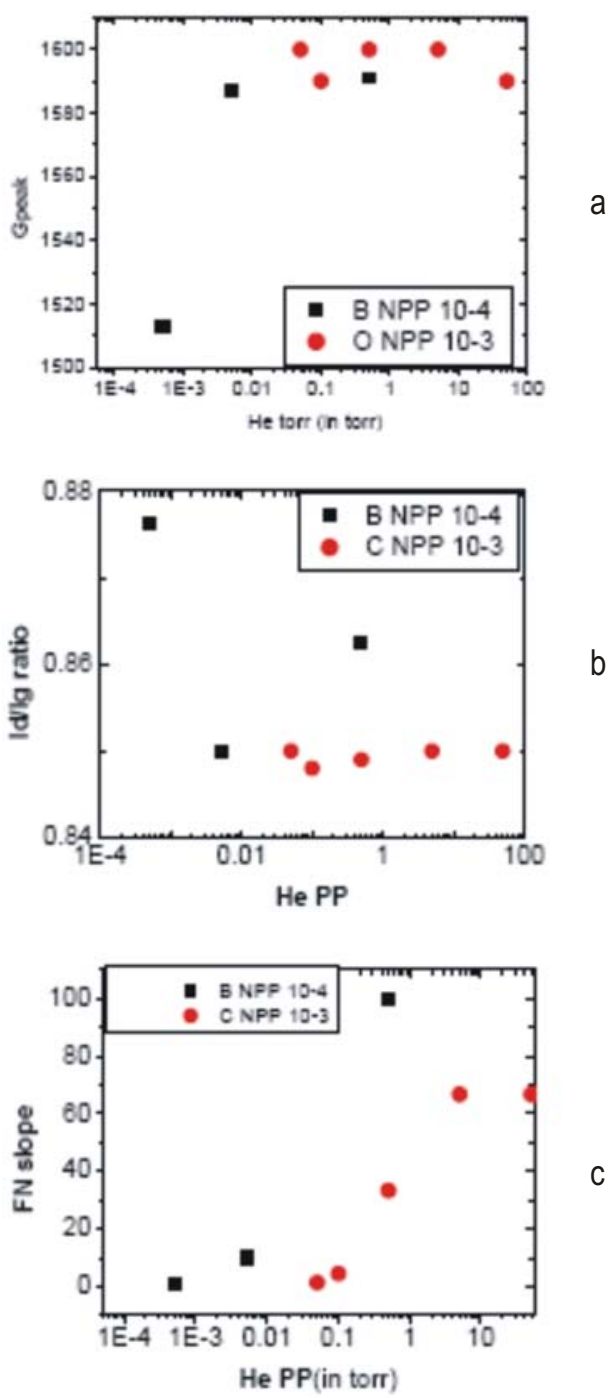

Fig 5 , Nanocluster samples grown with nitrogen partial pressure $10^{-3}$ Torr and $10^{-4}$ Torr with variation in helium(He) partial pressure, (a)G peak variation, (b) Variation of ID/IG ratio variation(c) Variation of FN slope

Shown in Fig. 4, 5 (a,b\&c) some of the relations based on the parameters derived from Raman response for nanocluster samples grown with nitrogen partial pressure $10^{-3}$ and $10^{-4}$ Torr with variation in helium partial pressure from $5 \times 10^{-4}$ torr to 50 torr. The large variation of Full Width Half Maximum(FWHM) under nitrogen partial pressure $10^{-4}$ when helium concentration changes correlates with dimensional variation as seen in SEM images. 
Shown in figure $5(\mathrm{a})$, is the variation of $\mathrm{G}$ peak position derived from Raman response for nanocluster samples grown with nitrogen partial pressure $10^{-3}$ and $10^{-4}$ Torr and varying helium partial pressure. A small shift in $G$ peak position under varying conditions of Helium partial pressure for a nitrogen partial pressure of $10^{-3}$ Torr, indicates that $\mathrm{sp}^{2}$ composition does not change much in this range of parameters study. However the SEM images show a greater range of change in the morphology and dimensions of the clusters. Whereas $\mathrm{sp}^{2}$ composition varies more drastically when nitrogen partial pressure is $10^{-4}$ Torr for varying Helium partial pressure. However the transition nature from $\mathrm{sp}^{3}$ type bonding to $\mathrm{sp}^{2}$ type bonding seems to be more pronounced at the nitrogen partial pressure of $10^{-4}$ Torr with in the samples studied. Figure $5(b)$ shows the variation of Id/lg ratio derived from Raman response for nanocluster samples grown with nitrogen partial pressure $10^{-3}$ and $10^{-4}$ Torr with variation of helium partial pressure. The small variation in $I_{d} I_{g}$ ratio in the above range of study, shows that the composition that nanocluser carbon grown is is predominantly $\mathrm{sp}^{2}$ or the clusters are highly graphitized in nature.

The correlation of FWHM of the G Peak and SEM images derived data indicate a more uniform cluster size and distribution possibility in case of nitrogen partial pressure $10^{-3}$ Torr. We have earlier reported field assisted electron emission at very low fields (1 to $10 \mathrm{~V} / \mathrm{\mu m}$ ) using these room temperature grown nanocluster carbon as cathodes $[8,11,14,19]$. From those field emission data the Fowler Nordheim slope (the basis to confirm that the electron emission is indeed from field assisted electron emission) was estimated. Shown in figure 5(c) is the variation of $\mathrm{FN}$ slope and with varying helium concentration during the growth of the nanoclsuter carbon. The study suggest the possibility of a possible optimum value of FN slope for a particular helium partial pressure for a given concentration of nitrogen which could give the best electron emission. In general higher the FN slopes better the field assisted electron emission characteristics. Shown in figure 6 is the image of glowing phosphor due to the excitation by electrons due to field assisted emission. The diode configuration was used in this experiment with anode consisting of patterned phosphorous screen and cathode being a nanostructured carbon film. The experiment was carried out under a vacuum of about $10^{-6}$ Torr. MIT 50 characters are displayed at an applied electric field of $2.6 \mathrm{~V} / \mu \mathrm{m}$.

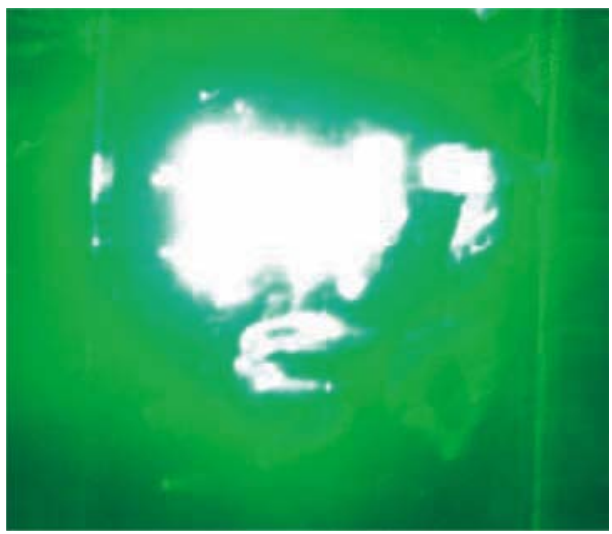

Fig 6. Patterned Field Emission based display

The study clearly suggested that with further study and optimization, the nanoluster carbon films grown at room temperature can be effectively tailored with any desired cluster dimension or surface morphology and electrical and electronics properties to make it a very attractive material for wide range of applications including surface engineering, sensors, nanoelectronics and vacuum nanoelectronic applications.

\section{CONCLUSION}

It was observed that under varying condition of helium and nitrogen nanocluster carbon films exhibit varying surface morphology. The surface morphology of the films grown, varied from atomic smooth surface to clusters with varying sizes (50-200 nm). The cluster size as well as composition varied more drastically at nitrogen partial pressure of $10^{-4}$ Torr. In the case of Nitrogen partial pressure of $10^{-3}$ Torr The dimension of the clusters varied from 50 to $200 \mathrm{~nm}$, but there was no appreciable change in the $\mathrm{sp}^{2} / \mathrm{sp}^{3}$ compositional ratio or the material was predominantly graphite like as estimated from Raman spectroscopy. There seems to be clear correlation between the structural and morphological properties and also the field assisted electron emission behaviour of the nanocarbons. Thus clearly indicating that with further optimization this room temperature grown nanocluster carbon thin films using the cathodic arc process could be a very interesting material for nanoelectronics applications.

\section{ACKNOWLEDGEMENT}

The authors would like to thank Innovation Center,Manipal Institute of Technology, Manipal University for providing some support for the nanotechnology research study. 


\section{REFERENCES}

[1] M.Terrones,A.Jorio,M.Endo,A.M.Rao,Y.A.Kim, T.Hayashi,H.Terrones,J.C.Charlier,G.Dresselha us and M.S.Dresselhaus, 2004. "New Directions in Nanotube Science," MaterialsToday,Oct,pp 30-45.

[2] Walt A De Heer, April,2004. "Nanotubes and Pursuit of Applications," MRS Bulletin.

[3] Niraj Sinha, JohnT, W.Yellow, June, 2005. "Carbon Nanotubes for biomedical Applications,"IEEE Transaction on Nanobioscience,Vol.4,No.2.

[4] Ta-Shan Chang, Ting-Chang Chang, Po-Tsun Liu, Tien-Shan Chang, Chun-Hao Tu, and FengSheng Yeh, Nov,2006. "Improvement of Hydrogenated Amorphous-Silicon TFT Performances With Low-k Siloxane-Based Hydrogen Silsesquioxane (HSQ) Passivation Layer," IEEE Electron Device Letters, Vol. 27, No. 11 .

[5] Niraj Sinha, Jiazhi Ma, and John T. W. Yeow 2006. "Carbon Nanotube-Based Sensors," Journal of Nanoscience and Nanotechnology ASP, Vol.6, 573-590.

[6] N.S.Xu and S.Ejaz Huq, 2005. "Novel cold cathode materials and applications," Material Science. \& Engg. R 48,47.

[7] B.S.Satyanarayana, 2001. "Room Temperature Grown Nanocarbon based multilayered Field Emitter Cathodes for Vacuum Microelectronics," $11^{\text {th }}$ IWPSD Conference Proceedings, ed V.Kumar \&P.K.Basu, p278.

[8] A.Hiraki , B.S.Satyanarayana, 2003. "Field emission from multilayered Carbon films consisting of nanoseeded Diamond and nanocluster carbon Deposited at Room temperature on glass substrate," IEICE, Vol. E 86-C,No.5.

[9] C.A.Spindt, 1968. J.App. Phys 39, 3504.

[10] B.S. Satyanarayana, A. Hart,W.I. Milne, and J.Robertson, 1997. "Field Emission from tetrahedral amorphous carbon," Phys. Letters, 71, p1430.

[11] B.S.Satyanarayana,J.Robertson, W.I.Milne, 2000. "Low Threshold Field Emission From Nanoclustered Carbon Grown by Cathodic Arc," J.App. Phys. 87, No.6,3126,
[12] A.C.Ferrari, B.S.Satyanrayana, P.Milani, E.Barborini, P.Piseri, J.Robertson, W.I.Milne, 1999. "Low Field electron emission from nanocluster carbon films grown using cluster assembled carbon process," Euro Phys.Lett, 46, 245.

[13] B.S.Satyanarayana, X.L.Peng, G.Adamapolous, J.Robertson, W.I. Milne, and T.W. Clyne, 2000. "Very Low Threshold Field Emission from Microcrystalline Diamond films grown using Hot Filament CVD Process," MRS Symp. Proc., Vol.621.

[14] B.S.Satyanarayana 2005. "The influence of $\mathrm{sp}^{3}$ bonded carbon on field emission from nanostructured $\mathrm{sp}^{2}$ bonded carbon films," IEEE, $18^{\text {th }}$ International Vacuum Nanoelectronics Conference Technical Digest, Oxford ,p219.

[15] B.S.Satyanarayana, B.Q.Wei, Y.Jung, G.Ramanath and P.M. A.Jayan , 2003. "Field Emission from aligned carbon nonotubes grown on pattererned oxide layers," $16^{\text {th }}$ IVMC Proceedings, P2-09.

[16] A.C.Ferarrari and J.Robertson, 2000. "Interpretation of Raman Spectra of Disordered and Amorphous carbon," Phy.Rev.B.61, 14095.

[17] Takao Yagi,Clare Hall, August 1999. "Electronic properties of amorphous carbon," Thesis report,University of Cambridge, Department of Engineering.

[18] O.S.Panwar,B.Deb,B.S.Sathanarayana, Mohd.AlimKhan, R.Bhattacharyya,A.K.Pal, 2004. "Characterization of as grown and nitrogen incorporated tetrahedral amorphous carbon films deposited by pulsed unfiltered cathodic vacuum are process," Thin Solid Films,Elsevier,

[19] B.S.Satyanarayana, 2005. "A comparison study of field assisted electron emission from nanocluster carbon films grown using a continuous and a pulsed cathodic arc process," IEEE $18^{\text {th }}$ International Vacuum Nanoelectronics Conference Technical Digest, Oxford.

[20] J.Robertson, 2002. "Diamond Like amorphous carbon," Materials Science and Engineering, $\mathrm{R}$ 37. 


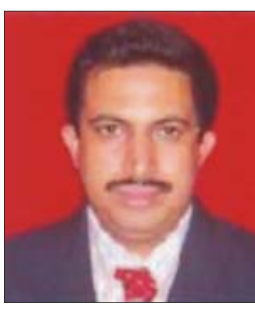

Niranjana.S, presently working as a Senior Grade Lecturer at Manipal Institute of Tech. (MIT),Manipal University, Manipal.He obtained his M.Tech Degree from Manipal Institute of Technology in the year 1998. He obtained B.E Degree from K.V.G. College of Engineering, Mangalore University in the year 1995. His interested areas includes :Micro/ nanoelectronics, Pattern recognition, Computer \& communication Networks, Programming and miniaturized system design.. Presented /published as aauthor/co-author for 17 research papers in national / international conferences.

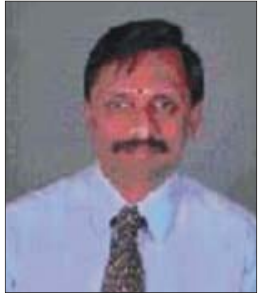

Dr. B. S. Satyanarayana holds PhD. Degree in Electrical Engineering from Cambridge University, UK. He has worked in Industry (Established and new ventures), R\&D Institutes and Academic Institutes for 22 years. Successfully implemented many projects from concept to proof of concept and also final projects for Industries. He has worked in India and abroad with collaborators in Industry, R\& D labs and research institutions from UK, Japan, US, Korea and Russia. He has over 50 research publications. The area of research interest include Nanotechnology, Novel electronics Material and Devices, Vacuum Nanoelectronics, Large area Microelectronics and Flexible Electronics, Flat Panel Displays, MEMS, Networks \& Sensors and Energy Harvesting and Energy Conversion Technologies. 